\title{
Spectroscopic observations of southern nearby galaxies ${ }^{\star}$
}

\author{
I. NGC 2442 \\ E. Bajaja ${ }^{1, \star \star}$, E. Agüero ${ }^{2, \star \star}$, and S. Paolantonio ${ }^{2}$ \\ 1 Instituto Argentino de Radioastronomía, C.C. 5, 1894 Villa Elisa, Prov. de Bs.As., Argentina \\ 2 Observatorio Astronómico de Córdoba, Laprida 854, 5000 Córdoba, Argentina
}

Received August 31, 1998; accepted January 4, 1999

\begin{abstract}
The galaxy NGC 2442 was observed with a REOSC spectrograph, installed in the $2.15 \mathrm{~m}$ CASLEO telescope, in order to derive galactic parameters from the observed optical lines and to compare them with the results of radioastronomical observations made in the continuum, at $843 \mathrm{MHz}$, with the MOST and in the CO lines with the SEST telescope. Recent publications allowed us to extend the comparison to results from interferometric observations of $\mathrm{H} \alpha$ and $\mathrm{HI} 21 \mathrm{~cm}$ lines and of the continuum at $1415 \mathrm{MHz}$. The long slit observations were made placing the 5.8 slit at six different positions on the optical image of the galaxy. The emission line intensity ratios at the nuclear region indicate that NGC 2442 is a LINER. The electron temperature and volume density are $T_{\mathrm{e}} \approx 14000 \mathrm{~K}$ and $N_{\mathrm{e}} \approx 530 \mathrm{~cm}^{-3}$, respectively. In contrast, a spectrum of a region $87^{\prime \prime}$ to the NE shows the typical characteristics of a $\mathrm{H}$ II region. In this case $T_{\mathrm{e}} \approx$ $6500 \mathrm{~K}$ and $N_{\mathrm{e}} \approx 10 \mathrm{~cm}^{-3}$. Good correlations between the distributions of intensities, velocity fields and rotation curves have been found for the optical and radio lines. It is shown that the three intensity peaks along the line at $\mathrm{PA}=40^{\circ}$ were not resolved by the observations at radio frequencies. The steep central rotation curve seen in $\mathrm{CO}$ has been confirmed and improved showing the existence of a disc or a ring, with a radius of 12.5 , rotating at $216 / \sin (i) \mathrm{km} \mathrm{s}^{-1}$. Two velocity components in three optical spectra obtained in the nuclear region, have been related to two small $\mathrm{H} \alpha$ regions close to the nucleus and to the central ring. Asymmetries in the distributions of the
\end{abstract}

Send offprint requests to: E. Bajaja,

e-mail: bajaja@irma.iar.unlp.edu.ar

* Based on observations made in the Complejo Astronómico El Leoncito, operated under agreement between the Consejo Nacional de Investigaciones Científicas y Técnicas de la República Argentina and the National Universities of La Plata, Córdoba and San Juan.

** Member of the Consejo Nacional de Investigaciones Científicas y Técnicas. emitting sources and irregularities in their velocity fields indicate the need of modelling the galaxy before any dynamical study is attempted.

Key words: galaxies: NGC 2442; kinematics and dynamics; nuclei; ISM

\section{Introduction}

NGC 2442 is a southern SAB(s)bc pec (de Vaucouleurs et al. 1991) galaxy belonging to a small group in Volans. A visual inspection of an optical image of the galaxy (Fig. 1) permits to appreciate that it is a very asymmetrical object, mainly due to the differences in the lengths and shapes of the northern and southern spiral arms. The main body consists of a central region from which two arms originate at each side of it and run along rather straight lines in the NE-SW direction (inner parts) till they change the direction, rather abruptly, starting the external parts of the arms. The inner parts are asymmetrical in the sense that the southern part is better defined and longer than the northern part. In the external parts the asymmetry appears in the opposite order: the northern arm is narrow, well defined and very extended towards the west while the southern arm is much shorter, broader and messy. Dust is prominent and can be seen projected everywhere against the luminous parts of the galaxy and sharing the characteristics described above for the optical appearance. In particular, the external part of the narrow northern arm is split into two parts along most of its extension by a narrower dust lane seen projected against it.

There have been several photometric observations of this galaxy in the past (Wegner 1979; Griersmith 1980; Peterson 1982; Baumgart \& Peterson 1986). More recently Ryder \& Dopita (1993) and Sersic \& Donzelli (1993) made more detailed photometric studies. Ryder \& Dopita (1993) 
made CCD images through narrow band $\mathrm{H} \alpha$ filters and a red continuum $(6676 \AA)$. Their $\mathrm{H} \alpha$ map displays very clearly the large number of $\mathrm{H}$ II regions and their nearly continuous distribution along the arms. Sersic \& Donzelli (1993) used photographic plates and a narrow $\mathrm{H} \alpha$ filter. They concluded that NGC 2442 is a rather normal barred spiral galaxy which should be classified as $\mathrm{SAB}(\mathrm{rs}) \mathrm{bc}$. They derived a distance modulus of $30.8 \mathrm{mag}$ and a total blue magnitude for the galaxy of $B_{\mathrm{T}}=-20.5 \mathrm{mag}$. The nucleus, according to Sersic \& Donzelli (1993), is elongated with a position angle (PA) which varies from $40^{\circ}$ at $5^{\prime \prime}$ to $60^{\circ}$ at $20^{\prime \prime}$ and the bar lies at a PA of $97^{\circ}$.

From spectroscopic observations, Shobbrock (1966) suggested that NGC 2442 might be a Seyfert or other emission type nucleus and Véron-Cetty \& Véron (1986) classified it as Seyfert-like galaxy. Recently, Mihos \& Bothun (1997) (MB97) and Houghton (1998) (H98) observed NGC 2442 in $\mathrm{H} \alpha$ with Fabry Perot instruments on the CTIO $1.5 \mathrm{~m}$ telescope and on the $3.9 \mathrm{~m}$ Anglo-Australian Telescope, repectively. MB97 found evidence in the nuclear region of emission from a central source and from a ring with a radius of $8^{\prime \prime}$ and an inclination of $69^{\circ}$. They suggest that a combination of Seyfert (central source) and starburst activity (ring) might be the cause for the confusion about the galaxy being a Syfert or a Seyfert-like. H98 detected less regions than MB97 (because of shorter integration times) but she was able to detect two small $\mathrm{H} \alpha$ emission regions, at both sides of the nucleus, separated by less than $5^{\prime \prime}$.

NGC 2442 has been observed in the H I $21 \mathrm{~cm}$ line, with single dishes, by Reif et al. (1982) and by Bajaja $\&$ Martin (1985). The low angular resolutions (13' and $30^{\prime}$, respectively) permitted to obtain only global velocity profiles which, nevertheless, provided information about the systemic velocities and about the $\mathrm{HI}$ and indicative masses. The observation of the ${ }^{12} \mathrm{CO}(1-0)$ line (Bajaja et al. 1995) with the SEST telescope, with an angular resolution of $43^{\prime \prime}$ and a velocity resolution of $7.2 \mathrm{~km} \mathrm{~s}^{-1}$, provided a more detailed picture of the distribution and velocity field of the molecular gas. In particular, the rotation curve showed a very steep velocity gradient at the center implying a fast rotation feature present there which, due to the angular resolution of the SEST, could not be properly resolved. The general distribution of the molecular gas over the main body of the galaxy is well correlated with the continuum emission at $843 \mathrm{MHz}$ mapped by Harnett (1984), with the Molonglo Observatory Synthesis Telescope (MOST), also with an angular resolution of $43^{\prime \prime}$. In both maps there is a N-S asymmetry, as seen in the optical image, and there are three main concentrations of emission, one at the center and one at each end of the main body, at the NE and the SW on the major axis. The spectral index derived by Harnett for this galaxy, on the basis of four frequencies between 408 and $5000 \mathrm{MHz}$, is $\alpha=-0.92 \pm 0.08$ which indicates the pre-eminence of the synchrotron emission over the thermal radiation.

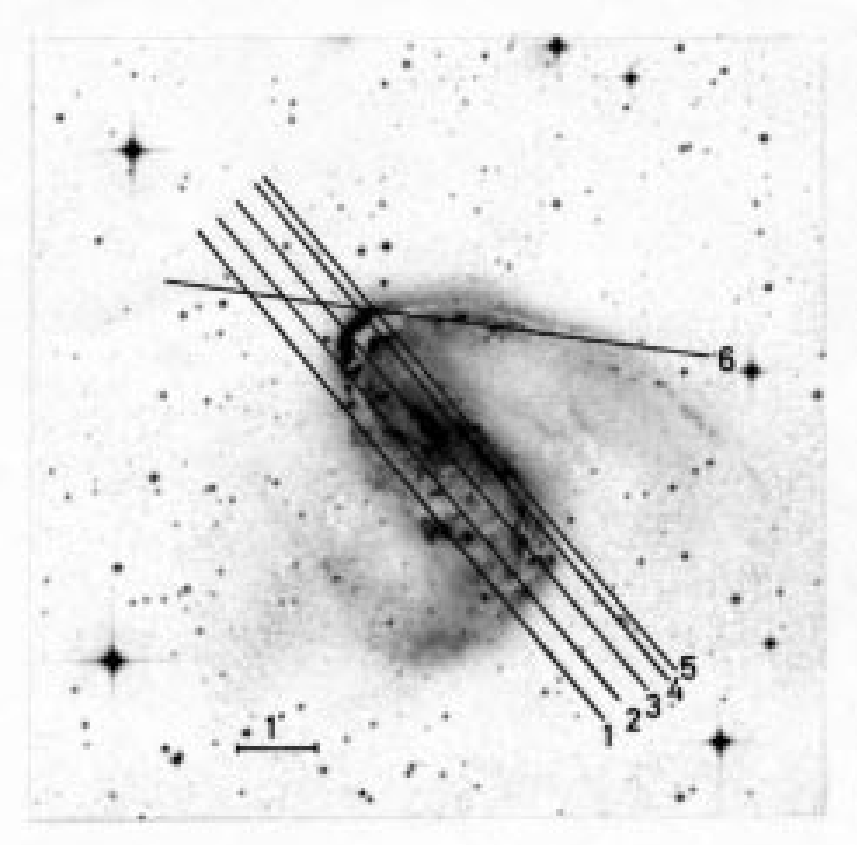

Fig. 1. Optical image of NGC 2442 from a SERC IIIa J plate obtained from the Digital Sky Survey (DSS). North is at the top and East to the left. The lines represent the positions of the slit: 1 to 5 at $\mathrm{PA}=40^{\circ}, 6$ at $\mathrm{PA}=79^{\circ}$

Besides the $\mathrm{H} \alpha$, H98 observed also the $\mathrm{H}$ i $21 \mathrm{~cm}$ line on NGC 2442 using the Australian Telescope Compact Array (ATCA). The channel maps were made with a velocity resolution of $\sim 16 \mathrm{~km} \mathrm{~s}^{-1}$ and a synthesised beam of $24^{\prime \prime} .6 \times$ $26^{\prime \prime} 2$. A $20 \mathrm{~cm}(1415 \mathrm{MHz})$ continuum map was also produced. The H I distribution and the continuum maps are similar except for the central region where the continuum emission is strong and the $\mathrm{HI}$ emission is weak. Also in this case both maps reproduce the asymmetries noticed at other wavelengths.

In the next two Sects. we describe our optical observations and the results and in the last Sect. we discuss these results and their comparison with the results of observations at other frequencies.

\section{Observations and reduction}

The spectroscopic observations were performed during dark time on the nights of February 24 to 26, 1995, using a REOSC spectrograph mounted on the $\mathrm{f} / 8.48$ Cassegrain focus of the CASLEO $2.15 \mathrm{~m}$ Ritchey-Chrétien telescope in El Leoncito (San Juan, Argentina). The seeing was between $2^{\prime \prime}$ and $3^{\prime \prime}$. The detector was a Tektronix CCD $1024 \times 102424 \mu \mathrm{m}$ pixels, corresponding each to $00^{\prime \prime} 26$. The exposures were obtained through a slit of $33^{\prime \prime} 3 \times 348^{\prime \prime}$, on the focal plane, at the six positions indicated in Fig. 1. Five are at $\mathrm{PA}=40^{\circ}$, which was assumed to be the PA of the line of nodes, and one along the narrow northern 
Table 1. Observations

\begin{tabular}{|ccc|}
\hline $\begin{array}{c}\text { Position }^{a} \\
\text { No. }\end{array}$ & $\begin{array}{c}\text { Lines } \\
\mathrm{mm}^{-1}\end{array}$ & $\begin{array}{c}\text { Exposure } \\
\text { min }\end{array}$ \\
\hline 1 & 1200 & 30 \\
2 & 1200 & 20 \\
3 & 300 & 60 \\
3 & 1200 & 40 \\
4 & 1200 & 60 \\
5 & 300 & 80 \\
6 & 1200 & 80 \\
\hline
\end{tabular}

${ }^{a}$ As given in Fig. 1.

arm, at a PA of $79^{\circ}$. All positions were referred to the center of the galaxy. Instead of shifting the slit for each position, the slit was positioned first on the center and then the telescope was moved in order to bring the slit to the required position.

In total, there were seven exposures, two of them using a 300 lines $\mathrm{mm}^{-1}$ grating at an angle of $4^{\circ} 50^{\prime}$, over the wavelength range of $\lambda \lambda 3500-7000$ (positions 3 and 5 in Fig. 1), and the other five with a 1200 lines $\mathrm{mm}^{-1}$ grating at an angle of $25^{\circ} 53^{\prime}$, covering the wavelength range of $\lambda \lambda 6200-6900$ (positions 1, 2, 3, 4, and 6 in Fig. 1). The dispersions were 125 and $32 \AA \mathrm{mm}^{-1}$, the reciprocal dispersions 3.1 and $0.76 \AA$ per pixel and the resolutions 10 and $2.5 \AA$ for the 300 and 1200 lines $\mathrm{mm}^{-1}$ gratings, respectively. The usual procedure was followed for the observations, including the observation of the flat field, the comparison spectra and the dark current. The exposures were, in general, of $20 \mathrm{~min}$ and repeated till the total time indicated in Table 1 was reached for each position. The spectra were corrected for atmospheric extinction and galactic reddening (Burstein \& Heiles 1984) and the flux was calibrated with stars from the catalog of Stone \& Baldwin (1982). Data reduction of the spectra was made employing standard methods of the IRAF (developed by NOAO) reduction package.

\section{Results}

The intensities of the emission lines present in the spectra (obtained with $125 \AA \mathrm{mm}^{-1}$ dispersion) of the nuclear region and of a region located at $87^{\prime \prime}$ towards the $\mathrm{NE}$ on the major axis, were derived fitting Gaussian curves to their profiles. In the nuclear spectrum the $\mathrm{H} \beta$ emission line is affected by the underlying $\mathrm{H} \beta$ absorption line, so Gaussian curves were interactively fitted to determine the absorption component and to isolate the $\mathrm{H} \beta$ emission. This procedure was verified by adding both, emission and absorption components, to reproduce the original profile. The internal reddening correction was carried out using the interstellar extinction curves given by Seaton (1979), assuming that the optical properties of the dust in
Table 2. Line Intensities relative to $\mathrm{H} \beta$

\begin{tabular}{|c|c|c|c|c|c|}
\hline \multirow[b]{2}{*}{ Ion } & \multirow[b]{2}{*}{$\begin{array}{l}\lambda \\
()\end{array}$} & \multicolumn{2}{|c|}{ Nucleus } & \multicolumn{2}{|c|}{$\mathrm{H}$ II region $\mathrm{NE}$} \\
\hline & & $\begin{array}{c}F \lambda / F \beta \\
I \lambda / I \beta\end{array}$ & $\overline{\sigma^{a}}$ & $\begin{array}{c}F \lambda / F \beta \\
I \lambda / I \beta\end{array}$ & $\sigma^{a}$ \\
\hline$[\mathrm{OII}]$ & 3727 & $\begin{array}{l}2.24 \\
2.27\end{array}$ & 0.13 & $\begin{array}{l}- \\
-\end{array}$ & - \\
\hline $\mathrm{H} \beta$ & 4861 & $\begin{array}{l}1.00 \\
1.00\end{array}$ & - & $\begin{array}{l}1.00 \\
1.00\end{array}$ & - \\
\hline [OIII] & 4959 & $\begin{array}{l}0.94 \\
0.94\end{array}$ & 0.06 & - & - \\
\hline [OIII] & 5007 & $\begin{array}{l}0.83 \\
0.83\end{array}$ & 0.05 & $\begin{array}{l}0.49 \\
0.46\end{array}$ & 0.09 \\
\hline$[\mathrm{NII}]$ & 5755 & $\begin{array}{l}0.23 \\
0.22\end{array}$ & 0.06 & - & - \\
\hline$[\mathrm{OI}]$ & 6300 & $\begin{array}{l}0.92 \\
0.91\end{array}$ & 0.05 & $\begin{array}{l}0.26 \\
0.16\end{array}$ & 0.02 \\
\hline$[\mathrm{NII}]$ & 6548 & $\begin{array}{l}2.53 \\
2.48\end{array}$ & 0.13 & $\begin{array}{l}0.70 \\
0.40\end{array}$ & 0.04 \\
\hline $\mathrm{H} \alpha$ & 6563 & $\begin{array}{l}3.16 \\
3.10\end{array}$ & 0.15 & $\begin{array}{l}4.98 \\
2.85\end{array}$ & 0.31 \\
\hline$[\mathrm{NII}]$ & 6584 & $\begin{array}{l}6.85 \\
6.71\end{array}$ & 0.31 & $\begin{array}{l}1.99 \\
1.13\end{array}$ & 0.14 \\
\hline$[\mathrm{SII}]$ & 6717 & $\begin{array}{l}2.50 \\
2.45\end{array}$ & 0.12 & $\begin{array}{l}0.99 \\
0.55\end{array}$ & 0.08 \\
\hline$[\mathrm{SII}]$ & 6731 & $\begin{array}{l}2.34 \\
2.30\end{array}$ & 0.11 & $\begin{array}{l}0.69 \\
0.38\end{array}$ & 0.06 \\
\hline $\begin{array}{c}c^{b} \\
\log I \beta^{c}\end{array}$ & & $\begin{array}{c}0.03 \\
-13.69\end{array}$ & & $\begin{array}{c}0.75 \\
-13.60\end{array}$ & \\
\hline
\end{tabular}

${ }^{a} \sigma$ is the absolute error of the $F \lambda / F \beta$ ratio,

${ }^{b} \mathrm{c}$ is the logarithmic extinction at $\mathrm{H} \beta$,

${ }^{c} I \beta$ is the flux corrected for reddening in erg $\mathrm{cm}^{-2} \mathrm{~s}^{-1}$.

NGC 2442 are similar to those in the Galaxy. The logarithmic extinction $c$, at $\mathrm{H} \beta$, was obtained using for the intrinsic ratio $\mathrm{H} \alpha / \mathrm{H} \beta$ the values 3.1 and 2.85 for the nucleus and for the region at the NE, respectively (Veilleux \& Osterbrock 1987). The measured and corrected line intensities $F \lambda$ and $I \lambda$, relative to $\mathrm{H} \beta=1.00$, are listed in Table 2 as well as the errors. These errors were estimated from the noise level around each line. The values of $c$ and of the corrected $\mathrm{H} \beta$ flux are given at the bottom of the table.

The mean radial velocities of all the spectra were derived measuring the centroids of Gaussian profiles centered on the emission lines [O I] $\lambda 6300,[\mathrm{~N}$ II] $\lambda \lambda 6548,6584, \mathrm{H} \alpha$, and [S II] $\lambda \lambda 6717,6731$ in the spectra obtained with a dispersion of $32 \AA \mathrm{mm}^{-1}$. The errors are estimated to be 3 to $26 \mathrm{~km} \mathrm{~s}^{-1}$, depending on the signal-to-noise ratio. The optical heliocentric radial velocities are shown in Fig. 5 superposed on the $\mathrm{CO}$ velocity field as obtained by Bajaja et al. (1995).

The spectrum at the nuclear region of the galaxy (Fig. 2) shows two velocity components, on each of the 


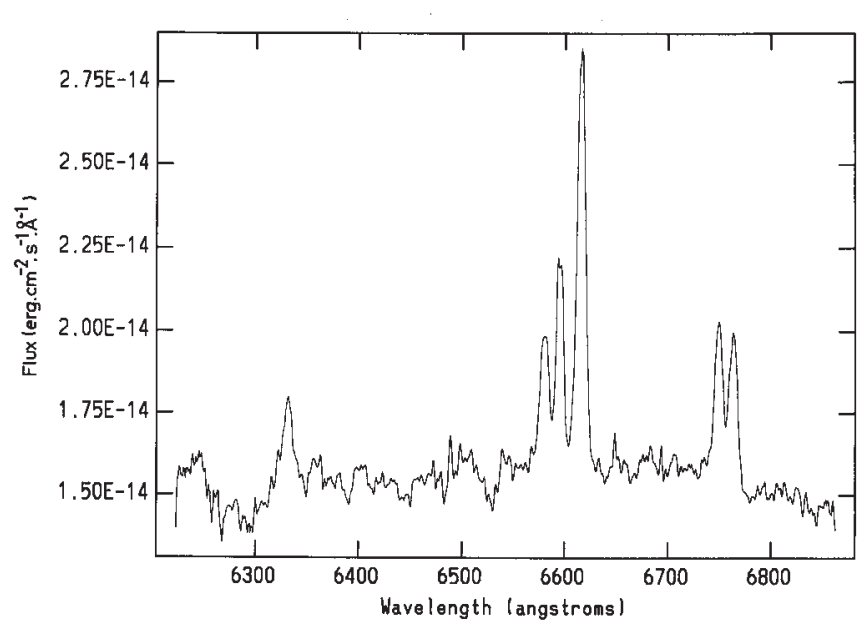

Fig. 2. Nuclear spectrum of NGC 2442. Dispersion $32 \AA \mathrm{mm}^{-1}$

prominent lines, with approximately the same intensity and a separation of about $145 \mathrm{~km} \mathrm{~s}^{-1}$.

\section{Discussion}

\subsection{Optical emission line intensity ratios}

The emission line intensity ratios at the nuclear region (Table 2) clearly indicate that it is a LINER (Osterbrock 1989), which is compatible with the suggestion of Shobbrock (1966) that this galaxy may be a Seyfert or other emission type. Véron-Cetty \& Véron (1986) classified it as a Seyfert-like galaxy, a designation for either a Seyfert or a LINER. However, from their measurements, they concluded that NGC 2442 is a $\mathrm{N}$ galaxy, with $\mathrm{H} \beta$ in absorption, because they found that $\mathrm{H} \alpha<1.2 \times[\mathrm{N} \mathrm{II}]$ $\lambda 6584$ but no other lines were present which would allow them to distinguish between a Seyfert 2 and a LINER. Being effectively a LINER, the dominant excitation mechanism should be photoionization by a continuous spectrum similar to but weaker than those present in Seyfert 2 galaxies (Osterbrock \& De Robertis 1985).

The electron temperature $T_{\mathrm{e}}$ and the density $N_{\mathrm{e}}$ at the nuclear region were derived from the $[\mathrm{N} \mathrm{II}](\lambda 6548+$ $\lambda 6584) / \lambda 5755$ and $[\mathrm{S} \mathrm{II}](\lambda 6717 / \lambda 6731)$ ratios, obtaining $T_{\mathrm{e}} \sim 14000 \mathrm{~K}$ and $N_{\mathrm{e}} \sim 530 \mathrm{~cm}^{-3}$, respectively, which are normal values for this type of objects. We might have here the situation mentioned by MB97 of seeing simultaneously the emission of a central point source (Seyfert) and of a star forming ring. Since the ring, in the $\mathrm{H} \alpha$ maps, appears quite thick and the inclination is about $69^{\circ}$ (as estimated by MB97), an appreciable amount of its emission should be present in the nuclear spectrum. We shall come back later to this possibility since we find also the central ring and we believe that our double peaked spectra in the nuclear region are produced by the two $\mathrm{H} \alpha$ sources detected by $\mathrm{H} 98$ and by the central ring.

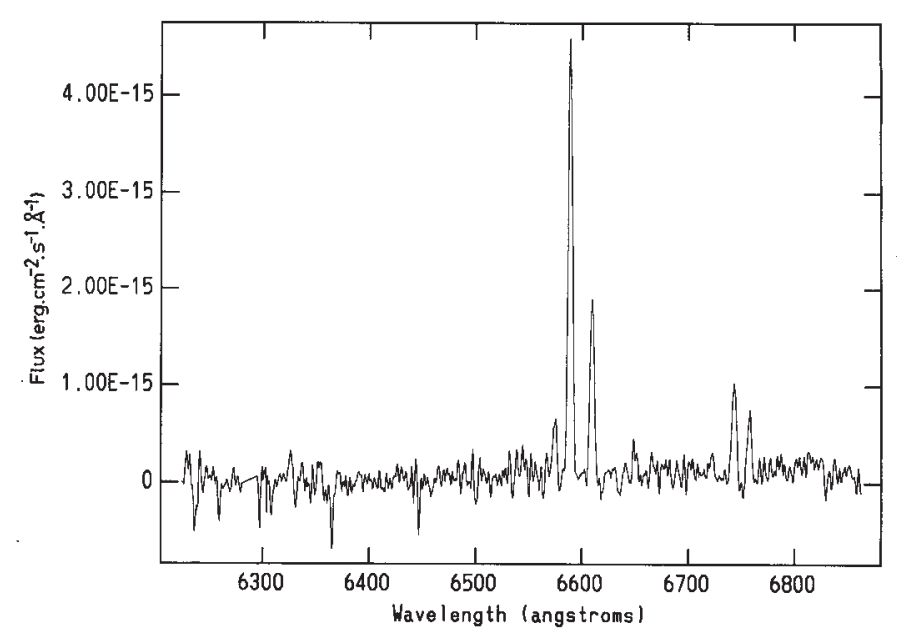

Fig. 3. Spectrum of an emission region located on the major axis at $87^{\prime \prime} \mathrm{NE}$ from the nucleus along $\mathrm{PA}=40^{\circ}$. Dispersion $32 \AA \mathrm{mm}^{-1}$

In Fig. 3 a spectrum of the region at the NE, mentioned above, is shown. Its spectral characteristics are typical of an H II region. The internal reddening is much higher than in the nuclear region. For this region the $N(\mathrm{O}) / N(\mathrm{H})$ and $N(\mathrm{~N}) / N(\mathrm{H})$ abundance ratios were obtained. The $N(\mathrm{O}) / N(\mathrm{H})$ abundance was derived from empirical calibrations from Edmunds \& Pagel (1984). Because the [O II $\lambda 3727$ line lies in a noisy zone of the spectrum, the [O II] $\lambda 3727 / \mathrm{H} \beta$ ratio was calculated from the first predicting equation given by McCall et al. (1985). Assuming $N(\mathrm{O}) / N(\mathrm{H})=\left(N\left(\mathrm{O}^{+}\right)+N\left(\mathrm{O}^{++}\right)\right) / N\left(\mathrm{H}^{+}\right)$ and $N(\mathrm{~N}) / N(\mathrm{O})=N\left(\mathrm{~N}^{+}\right) / N\left(\mathrm{O}^{+}\right)$, the nitrogen abundance is $N(\mathrm{~N}) / N(\mathrm{H})=\left(N\left(\mathrm{O}^{+}\right)+N\left(\mathrm{O}^{++}\right)\right) / N\left(\mathrm{O}^{+}\right)$ $\times\left(N\left(\mathrm{~N}^{+}\right) / N\left(\mathrm{H}^{+}\right)\right)$. The corresponding electron temperature was deduced from the equation for $N(\mathrm{O}) / N(\mathrm{H})$ searching for the required value of $T_{\mathrm{e}}$ for the previously derived $N(\mathrm{O}) / N(\mathrm{H})$ abundance. The resulting oxygen and nitrogen abundances, $N(\mathrm{O}) / N(\mathrm{H})=9.710^{-4}$ and $N(\mathrm{~N}) / N(\mathrm{H})=7.110^{-5}$, are 1.20 and 0.72 of the corresponding solar abundances. The ratio $N(\mathrm{~N}) / N(\mathrm{O})=0.07$ reflects a relative deficiency of $\mathrm{N}$ with respect to $\mathrm{O}$ but it is very close to usual values in galactic emission regions (Shaver et al. 1983). Its electron temperature and density: $T_{\mathrm{e}} \approx 6500 \mathrm{~K}$ and $N_{\mathrm{e}} \approx 10 \mathrm{~cm}^{-3}$, are slightly low but within the range of normal values.

\subsection{Correlation with other frequencies}

Our results consist of spectra at scattered points over NGC 2442, along the slit positions. They do not allow us to build contour maps for the comparison with the results of observations at other frequencies which did produce such maps. We can, however, make such a comparison along those slit positions. In particular, we were interested in the position at $\mathrm{PA}=40^{\circ}$ and through the nucleus, which 


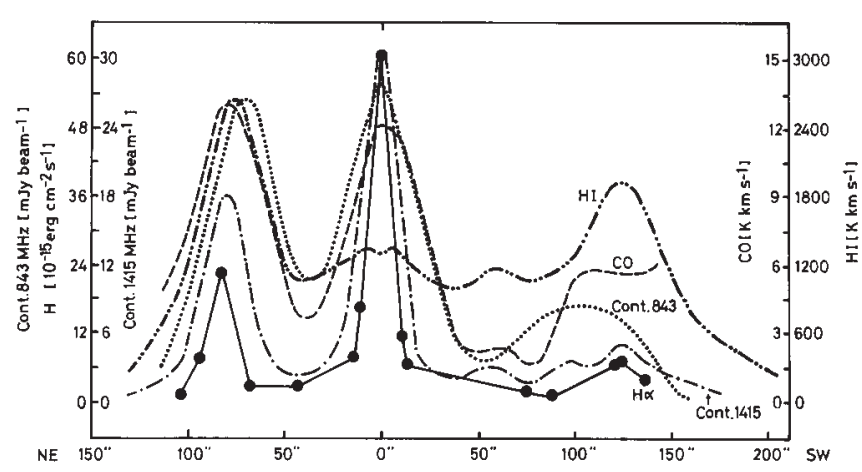

Fig. 4. Intensities as a function of the distance to the center, along a line at $\mathrm{PA}=40^{\circ}$, for the ${ }^{12} \mathrm{CO}(1-0)$ (Bajaja et al. 1995), $21 \mathrm{~cm} \mathrm{H} \mathrm{I} \mathrm{(H98)} \mathrm{and} \mathrm{H} \alpha$ (this paper) lines and for the continuum at $843 \mathrm{MHz}$ (Harnett 1984) and $1415 \mathrm{MHz}$ (H98)

we assumed to coincide with the line of nodes, because there are several important features along it. We shall start comparing the intensities.

\subsubsection{Intensities along $\mathrm{PA}=40^{\circ}$}

Figure 4 shows the flux densities of $\mathrm{H} \alpha$ (our results), in erg $\mathrm{cm}^{-2} \mathrm{~s}^{-1}$, and of the $843 \mathrm{MHz}$ (from Plate 2 of Harnett 1984) and $1415 \mathrm{MHz}$ continuum (from Fig. 5.4 of $\mathrm{H} 98)$, in mJy beam ${ }^{-1}$, and the areas of the ${ }^{12} \mathrm{CO}(1-0)$ (from Fig. 6b of Bajaja et al. 1995) and $21 \mathrm{~cm} \mathrm{H} \mathrm{I} \mathrm{(from}$ Fig. 5.7a of $\mathrm{H} 98$ ) velocity profiles, in $\mathrm{K} \mathrm{km} \mathrm{s}^{-1}$, along the line at $\mathrm{PA}=40^{\circ}$, as a function of the distance to the center. In spite of the different angular resolutions, which are evident in the widths of the central peaks, the correlation between the five components is clear. Except for $\mathrm{HI}$, which shows a rather flat curve in the central region, all the curves show three peaks, one at the center, one at about $80^{\prime \prime}$ towards the NE and a third one, much less defined, at about $125^{\prime \prime}$ towards the SW. The differences between the distances to the NE and SW peaks are another evidence for the asymmetry of the galaxy. The smallest width for the central source is shown by $\mathrm{H} \alpha$ with a FHMW of $\sim 16^{\prime \prime}$, which indicates that it is not resolved by the other observations. Differences in the positions of the peak centers at the NE, and probably at the SW, may be due to the fact that the data were taken from the figures and these may have untraceable scale errors, but the intensities are supposed to be correct.

In Table 3 are listed the peak values for the three regions (NE, Center and SW) and for the five types of emission. In order to be able to compare the emissions in the $\mathrm{CO}$ and $\mathrm{HI}$ lines, the profile areas were converted to column densities using for the conversion factors the values $310^{20}$ and $1.82310^{18}\left(\mathrm{~cm}^{2} \mathrm{~K} \mathrm{~km} \mathrm{~s}^{-1}\right)^{-1}$, respectively. For CO the conversion factor is the same as the one used by Bajaja et al. (1995) although there is not a general
Table 3. Peak values of intensities at different frequencies

\begin{tabular}{|ccccc|}
\hline Emission & Unit & NE & Center & SW \\
\hline Cont. $843 \mathrm{MHz}$ & mJy beam $^{-1}$ & 53 & 57 & {$[17]$} \\
Cont. $1415 \mathrm{MHz}$ & mJy beam $^{-1}$ & 18 & 30.8 & {$[5]$} \\
$\mathrm{H}_{2}$ & $10^{20} \mathrm{~cm}^{-2}$ & 39 & 37 & {$[18]$} \\
$\mathrm{H} \mathrm{I}$ & $10^{20} \mathrm{~cm}^{-2}$ & 48 & 25 & 26 \\
$\mathrm{H} \alpha$ & $10^{-15} \mathrm{erg} \mathrm{cm}^{-2} \mathrm{seg}^{-1}$ & 22.5 & 60.5 & {$[7]$} \\
\hline
\end{tabular}

Table 4. Intensity ratios

\begin{tabular}{|c|c|c|c|c|}
\hline Ratio & Unit & $\mathrm{NE}$ & Center & SW \\
\hline $843 \mathrm{MHz} / 1415 \mathrm{MHz}$ & & 2.94 & 18.5 & {$[3.4]$} \\
\hline Sp. index & & 2.08 & 1.19 & {$[2.4]$} \\
\hline $\mathrm{H}_{2} / \mathrm{H} \mathrm{I}$ & & 0.81 & 1.5 & {$[0.7]$} \\
\hline $\mathrm{H}_{2} / \mathrm{H} \alpha$ & $10^{35} \mathrm{~s} \mathrm{erg}^{-1}$ & 1.7 & 0.6 & {$[2.6]$} \\
\hline $\mathrm{H} \mathrm{I} / \mathrm{H} \alpha$ & $10^{35} \mathrm{~s} \mathrm{erg}^{-1}$ & 2.1 & 0.4 & {$[3.7]$} \\
\hline
\end{tabular}

agreement about the value that should be used. The continuum emissions are given in $\mathrm{mJy}_{\mathrm{beam}}{ }^{-1}$ so, for the comparison of these intensities, observing extended sources, the values should be corrected for the beams. In our case, however, we have seen that at both frequencies, $843 \mathrm{MHz}$ and $1415 \mathrm{MHz}$, the $\mathrm{NE}$ and central sources are not resolved. As for the southern part, the peaks in the curves are badly defined (values enclosed in brackets in Table 3) except for the H I whose peak has a FHMW similar to the one on the NE source.

Some of the ratios between the emission intensities are listed in Table 4. We used the flux densities quoted in Table 3, for the continuum emissions at 843 and $1415 \mathrm{MHz}$, to estimate the spectral indices which are also quoted in Table 4 . The ratio is larger at the center than at the NE and SW but all the spectral indices are indicating steep gradients in the flux densities for the three regions between both frequencies. As mentioned in Sect. 1, Harnett (1984) derived for the whole galaxy a spectral index value of $0.92 \pm 0.08$. All these values indicate a large pre-eminence of non-thermal radiation in the galaxy. The column density ratios for the lines also differ greatly in the three regions. The $\mathrm{H}_{2} / \mathrm{H}$ I column density ratio is higher at the center, as in the case of the continuum, but the ratios $\mathrm{H}_{2} / \mathrm{H} \alpha$ and $\mathrm{HI} / \mathrm{H} \alpha$ are much lower at the center. Evidently, the conditions for the conversion of atomic to molecular Hydrogen and from this to stars at the center are quite different from the conditions at the other regions.

\subsubsection{The rotation curve}

The velocities derived from our observations are indicated in Fig. 5 superposed on the $\mathrm{CO}$ velocity field (Fig. 6c in Bajaja et al. 1995). There is, in general, a good agreement between both if we take into account the errors. There are, however, differences which are due to the much 


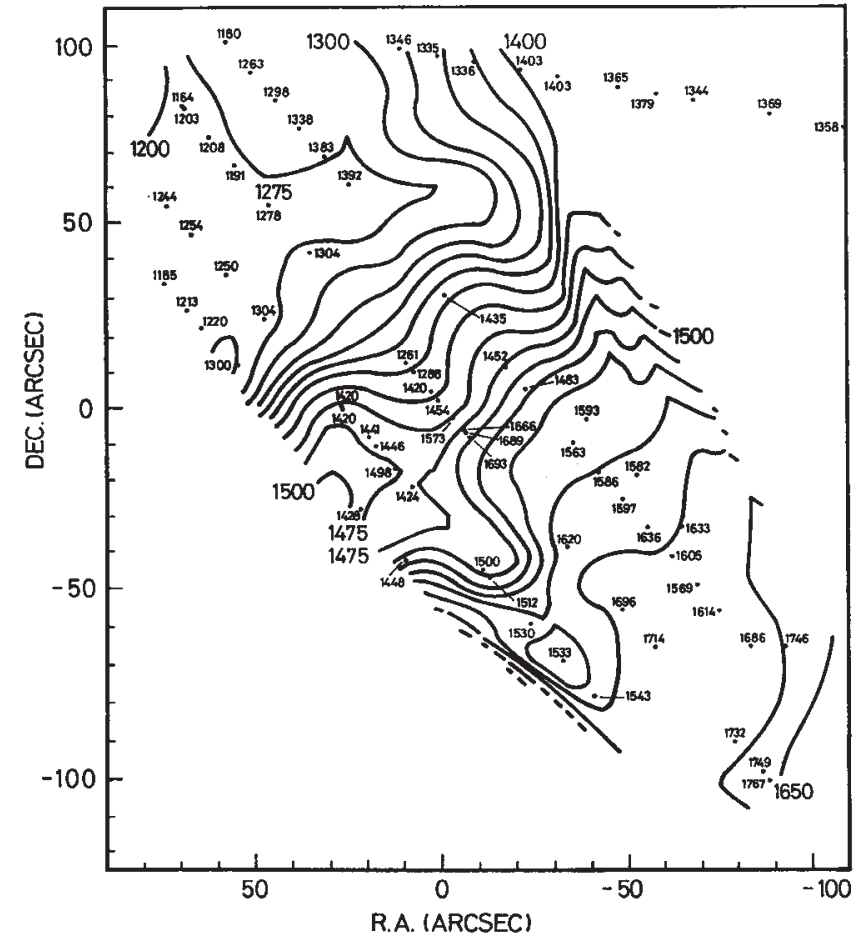

Fig. 5. Optical heliocentric radial velocities (small numbers at the positions indicated with dots) in $\mathrm{km} \mathrm{s}^{-1}$ and $\mathrm{CO}$ velocity field with contour levels in $\mathrm{km} \mathrm{s}^{-1}$ (large numbers), from Bajaja et al. (1995)

better angular resolution of the optical observations and to the different types of emission sources. This is particularly evident at the three regions mentioned in the previous section.

In Fig. 6 the optical velocities along the $\mathrm{PA}=40^{\circ}$ are shown superposed on the position-velocity diagram obtained with the CO spectra (Fig. 6d in Bajaja et al. 1995). The optical and the radio rotation curves, as derived from this figure, show similar velocity gradients in the central part in spite of the very different angular and velocity resolutions. The optical gradient, within $12^{\prime \prime} .5$ from the center, is $17 \mathrm{~km} \mathrm{~s}^{-1}$ per arcsec and it is approximately constant along its length. The velocities at the ends of this part of the velocity curve are 1262 and $1694 \mathrm{~km} \mathrm{~s}^{-1}$ and, from its symmetry, we adopt for the systemic velocity the value of $1478 \mathrm{~km} \mathrm{~s}^{-1}$ (the errors of these velocities are $\pm 4 \mathrm{~km} \mathrm{~s}^{-1}$ ). This feature may be associated either to a disk in solid rotation or to a ring rotating at $216 / \sin (i)$ $\mathrm{km} \mathrm{s}^{-1}$. Our spectra, along a line, do not allow us to have a two-dimensional picture of the object. MB97 derived similar parameters for a ring for which they obtained a radius of $8^{\prime \prime}$ and a rotation velocity of $220 / \sin (i)$, and for the systemic velocity a value of $1475 \mathrm{~km} \mathrm{~s}^{-1}$. All these values are practically the same as ours since the differences are within the errors. H98, however, derived for the systemic velocity, from the $\mathrm{H}_{\mathrm{I}}$ velocity field, the value of

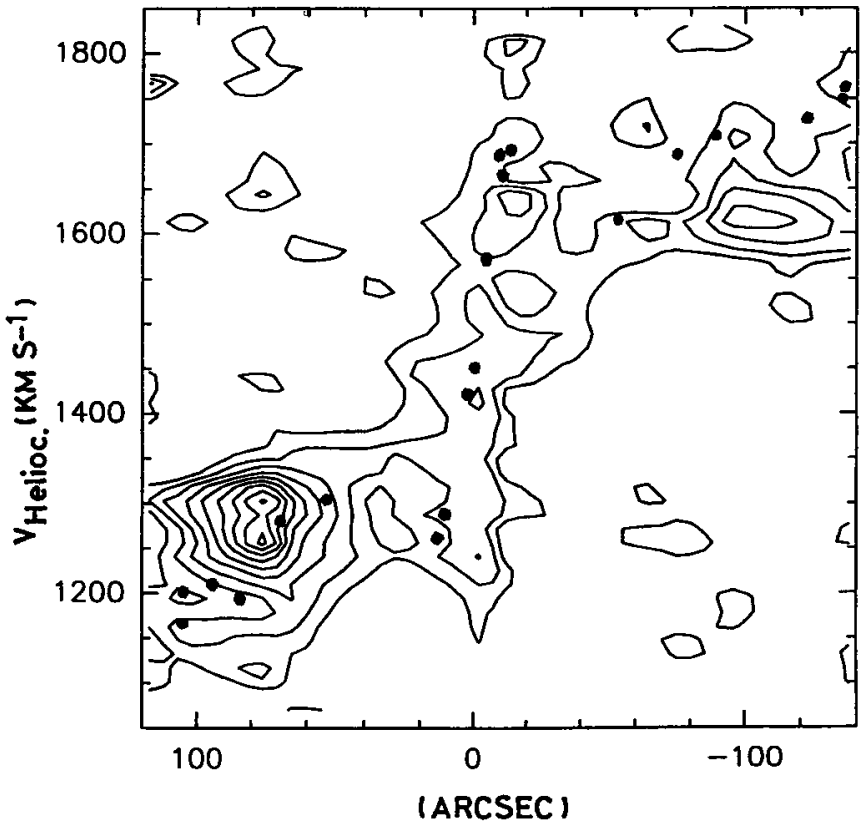

Fig. 6. Optical heliocentric radial velocities (dots) and CO velocity position diagram (from Bajaja et al. 1995) along the $\mathrm{PA}=40^{\circ}$. Contour levels in the latter are 12 to $108 \mathrm{mK}$, at intervals of $12 \mathrm{mK}$, of corrected antenna temperature

$1431 \mathrm{~km} \mathrm{~s}^{-1}$ which is much lower than the velocities mentioned above.

Our velocity for the galaxy, with respect to the centroid of the Local Group, is $1208 \mathrm{~km} \mathrm{~s}^{-1}$ so, using a Hubble constant $H_{0}=75 \mathrm{~km} \mathrm{~s}^{-1} \mathrm{Mpc}^{-1}$, the distance to NGC 2442 would be $16.1 \mathrm{Mpc}$ and the radius of the central disk or ring would be $\sim 1 \mathrm{kpc}$. MB97 estimated, from the geometry of the central ring, an inclination angle of $69^{\circ}$. Using this value the rotation velocity would be $231 \mathrm{~km} \mathrm{~s}^{-1}$ and the mass within the ring $\sim 1.210^{10} M_{\odot}$.

The optical rotation curve, as determined from our observations, extends up to $137^{\prime \prime}(10.7 \mathrm{kpc}$ at $16.1 \mathrm{Mpc})$ where the observed velocity, with respect to the center, is $275 \mathrm{~km} \mathrm{~s}^{-1}$. From the geometry of the optical image of NGC 2442 we may not assume a value as high as $69^{\circ}$ for the inclination angle of the outer parts of the galaxy. Although, most probably, it is not $24^{\circ}$ (Bajaja \& Martin 1985; Baumgart \& Peterson 1986) either, in the absence of a better value we adopt this one for the mass estimation. In consequence, the mass within $10.7 \mathrm{kpc}$ would be about $1110^{11} M_{\odot}$. From these values we may estimate then that, roughly, the mass within the ring is about $1 \%$ of the total mass, i.e. approximately proportional to the areas. The use of very different values of $i$ for difeerent distances from the center is a consequence of the large distortions present in NGC 2442. 


\subsubsection{Velocities in the nuclear region}

Our optical lines in the nuclear region show, as mentioned in the previous section, double velocity components. The spectra at $3^{\prime \prime} \mathrm{NE}$ and 5". $1 \mathrm{SW}$ also show two velocity components, with about the same separation as in the nucleus, but with some differences: a) the mean velocities of the spectra agree with the central rotation curve; b) the intensities of the velocity components at the NE are lower than at the nucleus but higher than at the SW, and c) while the intensities of both velocity components are approximately the same at the nucleus, they are different in the spectra at both sides of it and their intensity ratios are inverse respect to each other, i.e. in the spectrum at the NE the component with the shortest wavelength has the largest intensity and the opposite happens in the SW spectrum.

The explanation for these features has been searched for in the objects already mentioned: the two small $\mathrm{H} \alpha$ regions detected by $\mathrm{H} 98$ in the nuclear region and the central ring found by MB97 and by us. The $\mathrm{H} \alpha$ regions are about $2^{\prime \prime}$ in diameter and their peaks are separated by about 4. . 5 . The regions are, apparently, symmetrically positioned at both sides of the nucleus along a line at a $\mathrm{PA}$ of about $97^{\circ}$ so the angle between this line and our slit (at $\mathrm{PA}=40^{\circ}$ ) is $57^{\circ}$. The extension of the line seen by the $3^{\prime \prime} \cdot 3$ aperture is $4^{\prime \prime}$ so a large part of the emission of the $\mathrm{H} \alpha$ regions should have been detected and be present in the spectra on the nuclear region. The regions are displaced by 2 .' 2 along the slit. Our $\mathrm{H} \alpha$ spectrum at the nucleus of the galaxy encompasses both $\mathrm{H} \alpha$ regions symmetrically positioned but the two spectra at each side, to the NE and to the SW, cross them at different distances, i.e. with different weights.

About the central ring, the $\mathrm{H} \alpha$ maps of MB97 and of H98 show emission distributed over an oval region, centered in the nucleus and with the major axis at a PA of about $40^{\circ}$. If it is a ring then it is quite thick. The $\mathrm{H} \alpha$ velocity field of H98 shows clearly the rotation of this central feature and indicates that the PA of the line of nodes is the same as the PA of the major axis of the oval region. From the axis ratio of this region, MB97 derived an inclination angle of $69^{\circ}$. The two $\mathrm{H} \alpha$ regions and the points corresponding to our spectra in the nuclear region are inside this central fast rotating thick ring so, besides encompassing the two $\mathrm{H} \alpha$ regions, our three spectra should cover also the emission from the ring.

Therefore, if the intensities of both $\mathrm{H} \alpha$ regions are approximately the same, we would expect the following effects on our $\mathrm{H} \alpha$ spectra: a) the two velocity components at the nuclear position should be similar in intensity and displaced in velocity by the difference in the projected radial velocities of the two $\mathrm{H} \alpha$ regions; b) since the spectrum at the NE is closer to the two $\mathrm{H} \alpha$ regions its integrated line intensity should be larger than in the spectrum at the SW; c) in each spectrum, the velocity component correspond- ing to the closest $\mathrm{H} \alpha$ region, should be the strongest; d) the velocity difference for the two components should be approximately the same for the three spectra, and e) in each of our spectra, the material of the ring which is closer to its position will be seen with higher weight, pushing the mean velocity accordingly.

Qualitatively, the five effects are present in our spectra. Even quantitatively we have been able to reproduce the peak intensities on each of the three spectra assuming, for the sensitivity along the slit, a gaussian with a standard deviation $\sigma=33^{\prime \prime} 1 \pm 1^{\prime \prime}$. We might say then that the interpretation made above could be acceptable. There is a problem, however, with the central velocities given by $\mathrm{H} 98$ for the two $\mathrm{H} \alpha$ regions: 1367 and $1458 \mathrm{~km} \mathrm{~s}^{-1}$, with an average of $1412.5 \mathrm{~km} \mathrm{~s}^{-1}$ and a difference of $91 \mathrm{~km} \mathrm{~s}^{-1}$. We have, for the velocity components in the spectrum at the nuclear region, 1373 and $1518 \mathrm{~km} \mathrm{~s}^{-1}$, with an average of $1445.5 \mathrm{~km} \mathrm{~s}^{-1}$ and a difference of $145 \mathrm{~km} \mathrm{~s}^{-1}$. The separations between the component velocities differ by $54 \mathrm{~km} \mathrm{~s}^{-1}$ and the averages by $48 \mathrm{~km} \mathrm{~s}^{-1}$, differences which are much larger than the errors in our velocities. H98 also obtained, for the systemic velocity of the galaxy, $1431 \mathrm{~km} \mathrm{~s}^{-1}$ which is $47 \mathrm{~km} \mathrm{~s}^{-1}$ and $44 \mathrm{~km} \mathrm{~s}^{-1}$ lower than the values derived by us and by MB97, respectively. We do not know the reasons for these differences. We are inclined to think that our values are correct but the errors involved are much too high and strongly dependent on the procedure used for deriving the velocities.

There are further questions related to the two $\mathrm{H} \alpha$ regions detected by H98. Being inside the central ring, it is natural to assume that they participate of its rotation and that the velocity difference is due to the difference in the projected rotational velocities. The question is then: why both regions are along a line with a $\mathrm{PA}$ of $97^{\circ}$ and not at the edges of the ring, along the line of nodes? Furthermore, a difference of $145 \mathrm{~km} \mathrm{~s}^{-1}$ and a separation of $4^{\prime \prime} .5$ on a plane inclined $69^{\circ}$, would imply that the $\mathrm{H} \alpha$ regions are rotating with a velocity of about $345 \mathrm{~km} \mathrm{~s}^{-1}$. This velocity is $50 \%$ larger than the velocity of the ring. With a velocity separation of $91 \mathrm{~km} \mathrm{~s}^{-1}$ (as given by H98) that velocity would become $216 \mathrm{~km} \mathrm{~s}^{-1}$ but the distance to the center would be then twice the value measured in the $\mathrm{H} \alpha$ map of $\mathrm{H} 98$. The possibility that the two $\mathrm{H} \alpha$ regions are on the edges of a ring on a different plane, with the line of nodes at $97^{\circ}$ and with an unknown inclination angle, is not supported by the emission distribution in the $\mathrm{H} \alpha$ maps. There might be still another possibility: two $\mathrm{H} \alpha$ regions with expanding movements. It should be recalled that Sersic \& Donzelli (1993) specified for the bar also a PA of $97^{\circ}$. Might be there a connection? The questions are open.

We have to consider how the results described above could affect the previous conclusion, derived from the intensity ratios at the nuclear region, that it is a LINER. As was said then, this would imply that the dominant excitation mechanism is photoionization by a power-law 
continuum similar to but weaker than in Seyfert 2 galaxies. However, from the fact that our nuclear region spectrum sees the $\mathrm{H} \alpha$ regions, and that $\mathrm{H} \beta$ is also detected in absorption, it would be possible that this spectrum is the result of the integration of the emissions from a central point Seyfert source and from the star formation regions. This combination, which weakens the effect of a pure Seyfert source, would give as a result the characteristics of a LINER.

\subsubsection{Velocities in the disc}

The CO Velocity-Position diagram in Fig. 6 shows a tendency for double velocity components at both ends of the line at $\mathrm{PA}=40^{\circ}$. The optical velocities along this line, in those regions, seem to follow the $\mathrm{CO}$ components with the highest absolute velocities and the lowest intensities. It is not clear what this double velocity would mean. A warp could easily produce this kind of effect along the minor axis but it would have to be very strong to be visible along the major axis. More data are necessary for modelling it.

Figure 5 shows the velocities obtained for ten points along the external northern arm and it may be found that the velocities, at the far end, are between 1360 and $1370 \mathrm{~km} \mathrm{~s}^{-1}$. Since the errors are of the order of 3 to $10 \mathrm{~km} \mathrm{~s}^{-1}$, the conclusion is that these velocities are not compatible with a normally rotating inclined disc, with a $\mathrm{PA}$ for the line of nodes of $40^{\circ}$ and a systemic velocity of $1478 \mathrm{~km} \mathrm{~s}^{-1}$, because the points are then close to the minor axis. There might be several possible explanations for the abnormal velocities: a) a smaller PA; b) a velocity component normal to the galactic plane (warp?), c) a radial component along the plane (expansion?), etc, some of which might be ocurrying simultaneously. This is another evidence for the distortion of the galaxy. Almost certainly there are no single values for the PA of the line of nodes and for the inclination that may be applied to the whole galaxy.

Interaction with other galaxies might be playing a fundamental role in determining the matter distribution and the velocities on this galaxy. There are in fact several galaxies around that might be interacting with NGC 2442, but none of them is so close and with such a clear evidence of interaction as to be defined as the candidate. The consequence is that different authors suggest different galaxies: NGC 2434 (Elmegreen et al. 1992), AM 0738692 (MB97). H98 has considered several of the galaxies in the neighbourhood of NGC 2442 without pointing to any one in particular as the most probable candidate. The number of free parameters involved in the modelling of this galaxy requires many more data than those available for this work. The model, however, is largely necessary before any discussion about the dynamics of this galaxy may be attempted. MB97 considered the encounter with a nearby galaxy modelling the evolution of the stellar and gaseous components. H98 used two programs within the AIPS package, GAL and ROCUR, for deriving some basic parameters and then to make a three dimensional model of the galaxy using a constant inclination of $42^{\circ}$ and fitting the PA. Both models are important but still partial contributions to the knowledge of NGC 2442.

\section{Conclusions}

We made long slit spectroscopic observations of the galaxy NGC 2442 positioning the $3^{\prime \prime} 3 \times 348^{\prime \prime}$ slit on six different positions (Fig. 1), five at $\mathrm{PA}=40^{\circ}$ and one at $\mathrm{PA}=$ $79^{\circ}$. One of the five parallel positions was centered on the nucleus of the galaxy and the one at $\mathrm{PA}=79^{\circ}$, was positioned along the external northern arm. From the analysis of the reduced spectra, we arrived to the following conclusions:

1. The emission line intensity ratios at the nuclear region indicate that it is a LINER. The electron temperature $T_{\mathrm{e}}$ and the density $N_{\mathrm{e}}$ in this region are $T_{\mathrm{e}} \sim$ $14000 \mathrm{~K}$ and $N_{\mathrm{e}} \sim 530 \mathrm{~cm}^{-3}$, respectively, which are normal values for this type of objects.

2. The spectral characteristics of a region $87^{\prime \prime}$ to the $\mathrm{NE}$ are typical of an $\mathrm{H}$ II region. $N(\mathrm{O}) / N(\mathrm{H})=9.710^{-4}$ and $N(\mathrm{~N}) / N(\mathrm{H})=7.110^{-5}$. The ratio $N(\mathrm{~N}) / N(\mathrm{O})$ $=0.07$ is very close to usual values in galactic emission regions. $T_{\mathrm{e}} \approx 6500 \mathrm{~K}$ and $N_{\mathrm{e}} \approx 10 \mathrm{~cm}^{-3}$ are slightly low but within the range of normal values.

3. The intensities of $\mathrm{H} \alpha,{ }^{12} \mathrm{CO}(1-0), 21 \mathrm{~cm} \mathrm{H} \mathrm{I}$, and the continuum at 843 and $1420 \mathrm{MHz}$, along a line at $\mathrm{PA}=40^{\circ}$ and through the nucleus (Fig. 4), show all, except one, three peaks: one at the center, another one at about $80^{\prime \prime} \mathrm{NE}$ and the third one at about $125^{\prime \prime}$ SW. HI does not show the peak at the center. The sources at the positions of the peaks are not resolved. The intensities of the peaks and their ratios (Tables 3 and 4) show that the spectral indices for the two continuum frequencies and for the three peaks are large, indicating pre-eminence of non-thermal radiation. The $\mathrm{H}_{2} / \mathrm{HI}$ column density ratio at the center is higher than at the other two places while the ratios $\mathrm{H}_{2} / \mathrm{H} \alpha$ and $\mathrm{HI} / \mathrm{H} \alpha$ are much lower at the center, indicating different conditions for the conversion of atomic to molecular Hydrogen and molecular gas to stars.

4. There is a good correlation between the mean velocities of $\mathrm{H} \alpha$ and $\mathrm{CO}$ (Fig. 5). The velocity curve along the line at $\mathrm{PA}=40^{\circ}$ (assumed to be the line of nodes) shows a linear and steep velocity gradient within 12 '.5 from the center, with terminal velocities of 1262 and $1694 \mathrm{~km} \mathrm{~s}^{-1}$ (Fig. 6). This type of velocity curve may be caused by a fast rotating ring, in this case with a projected velocity of $216 \mathrm{~km} \mathrm{~s}^{-1}$. We adopt $1478 \mathrm{kms}^{-1}$ for the systemic heliocentric velocity and, using $H_{0}=75 \mathrm{kms}^{-1} \mathrm{Mpc}^{-1}$, a distance of 
16.1 Mpc. The central ring would have then a radius of $\sim 1 \mathrm{kpc}$. All these values are similar to those found by MB97. The optical velocity curve extends up to $137^{\prime \prime}$ (10.7 kpc at $16.1 \mathrm{Mpc}$ ) where the measured velocity is $275 \mathrm{~km} \mathrm{~s}^{-1}$ with respect to the center.

5. Using an inclination angle of $69^{\circ}$ (MB97), the rotation velocity of the central ring, at a radius of $12^{\prime \prime} .5$, would be $231 \mathrm{kms}^{-1}$ and the mass within its radius $\sim 1.210^{10} M_{\odot}$. The total mass within a radius of $137^{\prime \prime}$, assuming an inclination of $24^{\circ}$, would be $\sim 11$ $10^{11} M_{\odot}$. Therefore, the mass within the central ring would be about $1 \%$ of the estimated total mass, i.e. proportional to the areas.

6. The three optical spectra in the nuclear region, at $3^{\prime \prime}$ to the NE and $55^{\prime \prime} 1$ to the SW, show double velocity components separated by $145 \pm 4 \mathrm{~km} \mathrm{~s}^{-1}$. These components and their relative values might be explained by the presence of two $\mathrm{H} \alpha$ small regions (H98) separated 4.. 5 , symmetrically positioned at both sides of the nucleus and along a line at $\mathrm{PA} \approx 97^{\circ}$, and of the central ring. The velocities given by $\mathrm{H} 98$ for the central features do not agree neither with the velocities derived from our spectra nor with the systemic velocities found by us and by MB97. An explanation for these velocity differences should be found in the procedures for deriving them.

7. The LINER nature of the nuclear region could be the result of the integration of the emissions from a central point Seyfert source and from star formation regions.

8. The velocities along the external northern arm are incompatible with a disc in normal galactic rotation with the line of nodes at a $\mathrm{PA}=40^{\circ}$. Different parameters are required which must be obtained by modelling. In this modelling it is also necessary to include the effects of the probable interaction with some of the galaxies seen in the neighbourhood of the galaxy.

9. The final conclusion is that NGC 2442 requires still much work to be done to fully understand its morphology, dynamics and internal processes.

Acknowledgements. We acknowledge the cooperation of the Director and of the technical staff of the CASLEO observatory which made possible these observations. We also acknowledge the use of the CCD and data acquisition system supported under U.S. National Science Foundation grant AST-90-15827 to R.M. Rich. We are grateful to Sally Houghton for having access to her Thesis before publication.

\section{References}

Bajaja E., Martin M.C., 1985, AJ 90, 1783

Bajaja E., Wielebinski R., Reuter H.-P., Harnett J.J., Hummel E., 1995, A\&AS 114, 147

Baumgart C.W., Peterson C., 1986, PASP 98, 56

Burstein D., Heiles C., 1984, ApJS 54, 33

de Vaucouleurs G., de Vaucouleurs A., Corwin Jr. H.G., et al. 1991, Third Reference Catalog of Bright Galaxies. Springer Verlag

Edmunds M.J., Pagel B.E.J., 1984, MNRAS 211, 507

Elmegreen D.M., Elmegreen B.G., Bellin A.D., 1992, A\&A 257, 17

Griersmith D., 1980, AJ 85, 789

Harnett J.I., 1984, MNRAS 210, 13

Houghton S., 1998, Phd Thesis, University of New South Wales, Australia (H98)

McCall M.L., Rybski P.M., Shields G.A., 1985, ApJS 57, 1

Mihos J.C., Bothun G.D., 1997, ApJ 481, 741 (MB97)

Osterbrock D.E., 1989, in Astrophysics of Gaseous Nebulae and Active Galactic Nuclei. University Science Books, Mill Valley, CA

Osterbrock D.E., De Robertis M.M., 1985, PASP 97, 1129

Peterson C.J., 1982, PASP 94, 404

Reif K., Mebold U., Goss W.M., van Woerden H., Siegman B., 1982, A\&AS 50, 451

Ryder S.D., Dopita M.A., 1993, ApJS 88, 415

Seaton M.J., 1979, MNRAS 187, 73

Sersic J.L., Donzelli C., 1993, A\&AS 98, 21

Shaver P.A., McGee R.X., Newton L.M., Danks A.C., Pottash S.R., 1983, MNRAS 187, 73

Shobbrock R., 1966, MNRAS 131, 351

Stone R.P.S., Baldwin J.A., 1982, MNRAS 204, 347

Veilleux S., Osterbrock D.E., 1987, ApJS 63, 295

Véron-Cetty M.P., Véron P., 1986, A\&AS 66, 335

Wegner G., 1979, ApSS 60, 15 